\title{
SIMULAÇÃO MATEMÁTICA DO ESCOAMENTO BIFÁSICO NO DISTRIBUIDOR DE LINGOTAMENTO CONTÍNUO: ESTUDO DO EFEITO DA SUBSTITUIÇÃO DA BARRAGEM POR CORTINA DE GÁS
}

\author{
Leonardo Neves ' \\ Larissa de Barros Machado Vilela ${ }^{2}$ \\ Roberto Parreiras Tavares 3 \\ Ricardo Luiz Perez Teixeira ${ }^{4}$
}

\section{Resumo}

O lingotamento contínuo é um processo de solidificação de aço líquido onde o conhecimento de suas variáveis é de extrema importância para a produção de aços de qualidade. Este processo contém um reator denominado distribuidor que se localiza entre a panela e o molde e tem como função tradicional, distribuir o aço que sai da panela para o molde, mas também realiza outras importantes funções. Devido a essa importância no processo foi feito o estudo do escoamento do aço no distribuidor com duas configurações diferentes, sendo uma sem injeção de gás inerte e outra com injeção de gás inerte. Neste estudo foi analisado o efeito da substituição da barragem pela cortina de borbulhamento de gás, injetado no fundo do distribuidor, através da fluidodinâmica computacional. Para isto foi utilizado um software para a realização das simulações matemáticas. Foram encontradas diferentes curvas de distribuição de tempos de residência (curvas DTR), grandezas de turbulência e perfil de velocidade para as diferentes configurações.

Palavras-chave: Simulação matemática; Escoamento bifásico; Distribuidor de lingotamento contínuo; Cortina de injeção de gás.

\section{MATHEMATICAL SIMULATION OF TWO-PHASE FLOW INSIDE THE PHYSICAL MODEL OF CONTINUOUS CASTING TUNDISH: STUDY OF THE DAM SUBSTITUTION BY THE GAS CURTAIN}

\begin{abstract}
Continuous casting is a solidification process, in which the knowledge about its variables is very important in order to produce steel with good quality. The tundish distributes the steel coming from the ladle to the metallurgical mold as the traditional function, besides, it also has some other important functions. Because of its importance in the process, this work aim to carry out studies on the steel flow in the tundish with two different configurations, with and without inert gas injection. A Computational Fluid Dynamic (CFD) software were used to make the mathematical simulations making possible to note the difference in terms of the Residence Time Distribution curves (RTD curves), levels of turbulence and velocity profiles with or without inert gas injection.
\end{abstract}

Keywords: Mathematical simulation; Two-phase flow; Continuous casting tundish; Gas curtain.

\section{INTRODUÇÃO}

O lingotamento contínuo é um processo de solidificação de aço líquido muito importante se comparado ao lingotamento convencional devido à alta produtividade, excelente qualidade superficial e interna do aço. $O$ conhecimento de suas variáveis é de extrema importância para a produção de aços de qualidade. No lingotamento contínuo são basicamente utilizados três reatores metalúrgicos: a panela de aço, o distribuidor e o molde do lingotamento.

'Centro Federal de Educação Tecnológica de Minas Gerais - CEFET-MG, Belo Horizonte, MG, Brasil. E-mail: prof.leonardoneves@gmail.com.

'Universidade do Estado de Minas Gerais - UEMG, João Monlevade, MG, Brasil.

${ }^{3}$ Universidade Federal de Minas Gerais - UFMG, Belo Horizonte, MG, Brasil.

${ }^{4}$ Universidade Federal de Itajubá - UNIFEI, Itabira, MG, Brasil. 
O distribuidor se localiza entre a panela e o molde, tem como função tradicional, distribuir o aço que sai da panela para o molde. Devido à busca por melhorias na produção de aço, o distribuidor atualmente realiza várias funções metalúrgicas como: separação e flotação de inclusões, modificação da morfologia das inclusões formadas, ajuste fino da composição química, controle do superaquecimento e homogeneização térmica e química do aço. Modelagens matemáticas e físicas têm sido feitas a fim de estudar o comportamento do fluxo de aço dentro do distribuidor. De acordo com Szekely e llegbusi [I] e Schade et al. [2], o distribuidor contem três zonas distintas de fluxo, definidas como zona de fluxo pistonado, zona de mistura perfeita e zona de volume morto. Segundo Singh e Koria [3], há outra zona de fluxo no distribuidor denominada "curto-circuito". As condições hidrodinâmicas, que o distribuidor deve satisfazer, são mínimo volume morto (com o propósito de minimizar a segregação e a perda de temperatura do aço líquido no distribuidor), máxima fração de volume pistonado (para favorecer a remoção de inclusões não-metálicas), máxima região de mistura perfeita (para favorecer a coalescência das inclusões não-metálicas de pequenas dimensões), mínimo volume de "curto-circuito" (para inibir o arraste das inclusões não-metálicas do aço líquido) e faixa mínima de variação dos tempos de residências do metal líquido. Este reator pode apresentar formas variadas e modificadores de fluxo que auxiliam no controle do processo de lingotamento com a finalidade de produzir um aço de alta qualidade e/ou aumento da produção. Exemplos desses modificadores são: diques, barragens, injeção de gases, inibidores de turbulência dentre outros. Estes modificadores de fluxo são alternativas para a otimização do projeto do distribuidor e, consequentemente, do escoamento do metal líquido. A injeção de gás inerte pode trazer muitos benefícios ao distribuidor. $O$ gás, injetado no fundo do distribuidor, tem como principal objetivo a flotação de inclusões não-metálicas do aço líquido. Ele pode ser também injetado em regiões pontuais visando à redução de zonas de volume morto e assim contribuindo para homogeneização térmica e química do aço. A cortina de gás inerte pode servir para diminuir a zona de "curto-circuito", aumentando o tempo mínimo de residência do aço no distribuidor. Zhong et al. [4] e Cwudzinski [5] utilizaram diferentes configurações de dispositivos combinados com a injeção de gás inerte em seus estudos. O presente trabalho teve como objetivo estudar - efeito da substituição da barragem pela cortina de gás no escoamento do aço no distribuidor.

\section{MATERIAIS E MÉTODOS}

As dimensões do modelo físico utilizado na simulação computacional são apresentadas na Figura I. A Tabela I mostra as características do sistema de injeção de gás como: dimensão, quantidade e vazão dos bicos de injeção utilizados. Todo estudo foi baseado em análises dos

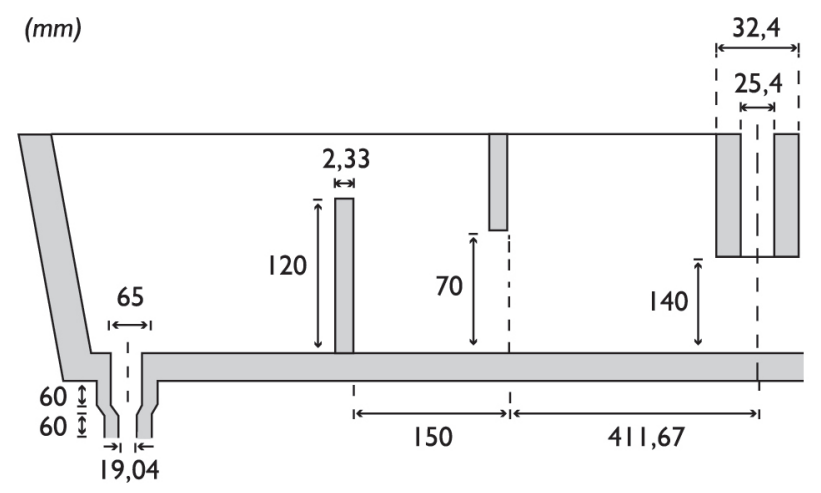

Figura I. Dimensões do distribuidor utilizado nas simulações matemáticas.

Tabela I. Característica do sistema de injeção de gases utilizados no distribuidor

\begin{tabular}{cc}
\hline Parâmetros & Injeção de gás \\
\hline - Injeção de gás no fundo: & \\
vazão de gás $(1 / \mathrm{min})$ & 2 \\
número de bicos & 4 \\
diâmetro dos bicos $(\mathrm{mm})$ & $\mathrm{I}$ \\
\hline
\end{tabular}

estudos da modelagem física e matemática do distribuidor desenvolvida por Neves [6].

No modelo computacional foram utilizadas as condições empregadas nos experimentos com o modelo físico do distribuidor. Para simular o aço líquido é utilizada água, pois a viscosidade cinemática da água na temperatura ambiente $\left(25^{\circ} \mathrm{C}\right)$ é $1,00 \times 10^{-6} \mathrm{~m}^{2} / \mathrm{s}$ aproximadamente igual a do aço a $1.600^{\circ} \mathrm{C}$ que é de $9,13 \times 10^{-7} \mathrm{~m}^{2} / \mathrm{s}$.

\section{I Métodos Matemáticos}

Para simular o escoamento turbulento foi utilizado o modelo do tipo k- $\varepsilon$. Foi utilizado o modelo de Grace para simular a força de arraste entre o gás e o líquido. $O$ critério de convergência, RMS, utilizado para declarar convergência da solução das equações de conservação foi de $10^{-6}$. A modelagem matemática do escoamento do aço foi feita para o estudo das condições fluidodinâmicas no distribuidor de lingotamento contínuo. Foram realizadas simulações com diferentes configurações do reator considerando o escoamento tridimensional bifásico ar/água. $O$ estudo foi realizado por meio do software ANSYS CFX. Utilizando de técnicas computacionais, este trabalho analisou principalmente os seguintes aspectos:

- Efeito do escoamento ar/água no modelo físico no distribuidor com os modificadores de fluxo dique e barragem sem injeção de gás;

- Efeito do escoamento ar/água no modelo físico do distribuidor com dique e injeção de gás no lugar da barragem;

- Curvas DTR, grandezas de turbulência e perfil de velocidade. 
As condições de contorno do modelo matemático foram estabelecidas de acordo com as condições físicas nos contornos do domínio do distribuidor. A Figura 2 mostra uma vista do domínio de simulação e as condições propostas para as configurações de distribuidor analisadas. As condições de contorno no distribuidor foram:

- Paredes: foi considerada a condição de não escorregamento. Para os gases, foi assumida a condição de escorregamento livre ("free slip");

- Bicos de injeção: foi considerada a entrada de gases. A vazão mássica de entrada de ar no modelo computacional foi de $3,95 \times 10^{-5} \mathrm{~kg} / \mathrm{s}$ (2 NI/min);

- Superfície livre do distribuidor: Foi assumida como uma condição de escorregamento livre. Para o sistema com injeção de gás, a superfície livre foi considerada como uma condição de saída dos gases, ou condição de desgaseificação, que permite que as bolhas do gás injetado saia do sistema ao atingirem esta superfície, e para a fase líquida foi assumida a condição de escorregamento livre;

- Simetria: foi considerada a condição de simetria de I/4 do equipamento nas simulações para ganho computacional.

\subsection{Abordagem Euleriana-Euleriana}

Segundo Paladino [7] há várias classificações para modelos multifásicos. Uma das mais utilizadas na literatura classifica o modelo em abordagem Euleriana-Euleriana. Nesta abordagem, cada fase tem seu próprio campo de variáveis (pressão, temperatura etc.), cada uma acoplada pelos modelos de transferência de quantidade de movimento, energia e massa. Os modelos de transferência mais simples assumem uma divisão no campo de pressão.

A equação de continuidade para uma fase $\alpha$ é expressa por (Equação I):

$$
\frac{\partial}{\partial t}\left(r_{\alpha} \rho_{\alpha}\right)+\nabla\left(r_{\alpha} \rho_{\alpha} u_{\alpha}\right)=\sum_{\beta=1}^{N}\left(M_{\alpha \beta}-M_{\beta \alpha}\right)
$$

em que:

$r_{\alpha}=$ fração de volume da fase $\alpha$;

$\rho_{\alpha}=$ densidade da fase $\alpha$;

$u_{\alpha}=$ velocidade da fase $\alpha$;

$N=$ número total de fases;

$M_{\alpha \beta}=$ taxa de transferência de massa da fase $\alpha$ para a fase $\beta$; $M_{\beta \alpha}=$ taxa de transferência de massa da fase $\beta$ para a fase $\alpha$.

\section{Entrada}

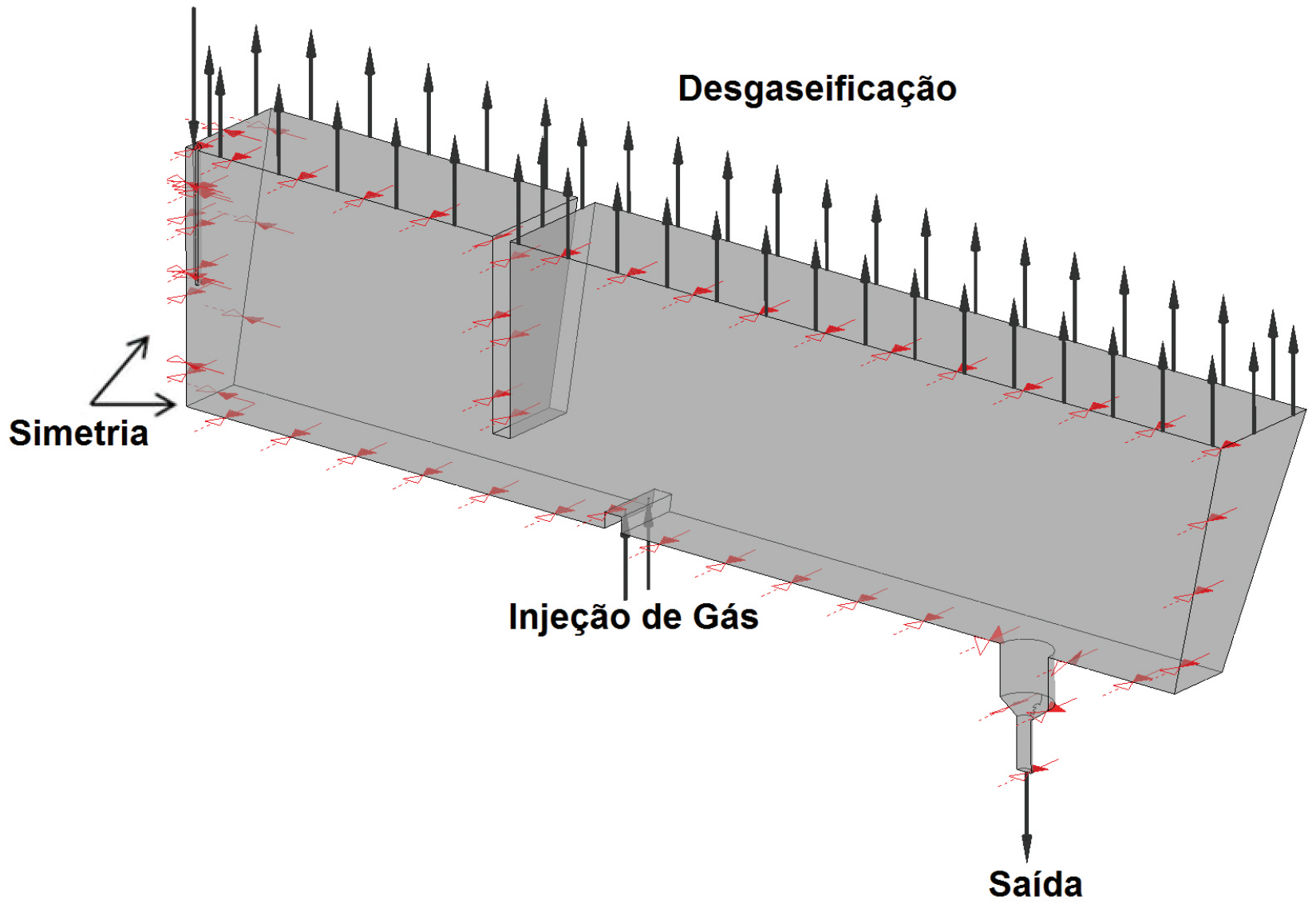

Figura 2. Domínio do distribuidor utilizado nas simulações. 
A soma das frações de volumes das fases é unitária (Equação 2):

$$
\sum_{\propto} r_{\infty}=1
$$

A equação de conservação de quantidade de movimento na direção x pode ser expressa por (Equação 3):

$$
\frac{\partial\left(\rho_{\alpha} r_{\alpha} u_{\alpha}\right)}{\partial t}+\frac{\partial\left(\rho_{\alpha} r_{\alpha} u_{\alpha} u_{\alpha}\right)}{\partial x}=-r_{\alpha} \cdot \frac{\partial P}{\partial x}+\frac{\partial\left(r_{\alpha} \tau_{\alpha}\right)}{\partial x}+r_{\alpha} \rho_{\alpha} g+S_{M \alpha}+M_{I \alpha}
$$

sendo (Equações 4 e 5):

$$
\begin{gathered}
S_{M \alpha}=F_{D}+F_{L}+F_{M}+F_{T}+F_{W} \\
M_{I \alpha}=\sum_{\beta=1}^{N}\left(M_{\alpha \beta} \cdot u_{\beta}-M_{\beta \alpha} \cdot u_{\alpha}\right)
\end{gathered}
$$

em que:

$r_{\alpha}=$ fração da fase $\alpha$;

$\rho_{\alpha}=$ densidade da fase $\alpha\left(\mathrm{kg} / \mathrm{m}^{3}\right)$;

$u_{\alpha}=$ velocidade da fase $\alpha(\mathrm{m} / \mathrm{s})$;

$u_{\beta}=$ velocidade da fase $\beta(\mathrm{m} / \mathrm{s})$;

$P=$ pressão $(\mathrm{Pa})$;

$\tau_{\alpha}=$ tensão cisalhamento que atua na fase $\alpha\left(\mathrm{kg} / \mathrm{m}^{3}\right)$;

$\mathrm{g}=$ aceleração da gravidade $\left(\mathrm{m} / \mathrm{s}^{2}\right)$.

O termo $S$ é a soma das forças interfaciais de arraste, $F_{D}$ ("drag force"), sustentação $F_{L}$ ("lift force"), massa virtual, $F_{M}$ ("virtual mass force") dispersão turbulenta, $F_{T}$ ("turbulent dispersion force"), lubrificação da parede, $F_{w}$ ("wall lubrication force") e a transferência de quantidade de movimento associada com a transferência de massa $M_{1}$ descreve as forças que atuam na fase $\alpha$ devido à presença da fase $\beta$.

As forças que atuam em uma fase são de mesma magnitude e em sentido oposto às que atuam na outra fase, logo o somatório das forças entre as fases se anula (Equação 6).

$$
M_{\alpha \beta} u_{\beta}=-M_{\beta \alpha} u_{\alpha}=\sum_{\alpha} M_{\alpha} U=0
$$

A quantidade de movimento transportada entre as fases é devido às forças de arraste e à transferência de massa.

\subsection{Curvas de Distribuição de Tempos de Residência}

Para a caracterização do fluxo do distribuidor foi utilizada a técnica proposta por Sahai e Emi [8]. A técnica de adição de traçador (normalmente um sal) mais adotada é a de injeção de pulso ("pulse-input") e os resultados obtidos podem ser representados graficamente utilizando concentração e tempo adimensionais. A curva de distribuição do tempo de residência ou curva normalizada é uma curva da concentração adimensional do sal em relação ao tempo adimensional de modo que a área sob a curva seja unitária. A injeção em pulso permite obter a curva DTR. Esta curva permite avaliar os aspectos do escoamento do aço no interior do reator e determinar os volumes das zonas de escoamento pistonado, mistura perfeita e volume morto. A concentração e o tempo adimensionais são determinados pelas Equações 7 e 8 :

$$
\begin{aligned}
& C=\frac{c}{q / V_{\text {dist }}} \\
& \theta=\frac{t}{t_{\text {teórico }}}
\end{aligned}
$$

em que:

$\mathrm{C}=$ concentração adimensional;

$c=$ concentração de traçador na saída a cada instante $t$

$\left(\mathrm{kg} / \mathrm{m}^{3}\right)$;

$\mathrm{q}=$ quantidade de traçador injetado $(\mathrm{kg})$;

$\mathrm{V}_{\text {dist }}=$ volume do distribuidor $\left(\mathrm{m}^{3}\right)$;

$\theta=$ tempo adimensional;

$t=$ tempo $(\mathrm{s})$;

$t_{\text {térico }}=$ tempo de residência nominal do reator (s).

Foram utilizados também o tempo mínimo de residência, $t_{\min }$, que representa $\circ$ tempo de início de saída do distribuidor, o tempo máximo, $\mathrm{t}_{\text {máx }}$, representando o tempo de ocorrência da concentração máxima na saída do distribuidor e $o$ tempo de residência médio, $t_{\text {médio }}$, que foi calculado pela Equação 9.

$$
t_{\text {médio }}=\frac{\sum_{t=0}^{t=2 . t_{\text {teórico }}} C_{i} t_{i} \Delta t_{i}}{\sum_{t=0}^{t=2 . t_{\text {teorico }}} C_{i} \Delta_{i}}
$$

em que:

$t_{\text {médio }}=$ tempo de residência médio real (s);

$t_{\text {teórico }}=$ tempo teórico de residência $(\mathrm{s})$;

$C_{i}=$ concentração do traçador em um tempo t.

$t_{i}=$ tempo de residência $\mathrm{i}(\mathrm{s})$;

$\Delta \mathrm{t}_{i}=$ variação de tempo do instante inicial até o instante i (s);

\section{RESULTADOS E DISCUSSÃO}

A análise de independência de malha para o modelo matemático sem injeção de gás foi feita utilizando as malhas de 168.467, 249.784, 290.752 e 458.679 elementos de volume. Para o modelo matemático com injeção de gás foram utilizadas as malhas de 304.074, 453.492 e 556.563 elementos de volume. Para a escolha da melhor malha foi utilizada a curva DTR das simulações e encontrou-se para o modelo sem injeção e com injeção de gás as malhas de 290.752 e 304.074 respectivamente. A curva DTR para ambas configurações está representada na Figura 3. Nota-se que para a configuração com a utilização da cortina de gás a curva DTR se torna mais suavizada. A Tabela 2 apresenta os resultados da configuração com e sem injeção de gás. Com o emprego da injeção de gás, observa-se que a fração de fluxo pistonado teve queda e o tempo médio real de residência diminuiu. Nota-se também, que houve um aumento na fração de volume morto com a injeção de gás e que ocorreu um aumento na fração do volume de mistura. A Figura 4 mostra o campo de vetores de velocidade a partir de um corte longitudinal para as configurações sem e com injeção de ar.

A distribuição de velocidade é parecida para as duas configurações utilizadas até o dique. A partir deste 
Tabela 2. Valores calculados para as configurações com e sem injeção de gás

\begin{tabular}{lccccccc}
\hline & $\mathbf{t}_{\min }(\mathbf{s})$ & $\mathbf{t}_{\text {máx }}(\mathbf{s})$ & $\mathbf{C}_{\mathbf{n} \text {, máx }}$ & $\mathbf{t}_{\text {médio }}(\mathbf{s})$ & $\mathbf{V}_{\text {morto }} / \mathbf{V}$ & $\mathbf{V}_{\text {pistonado }} / \mathbf{V}$ & $\mathbf{V}_{\text {mistura }} / \mathbf{V}$ \\
\hline Sem injeção & 38,34 & 127,8 & 1,14 & 172,15 & 0,17 & 0,11 & 0,72 \\
Com injeção & 8,52 & 115,0 & 0,82 & 161,68 & 0,22 & 0,04 & 0,74 \\
\hline
\end{tabular}

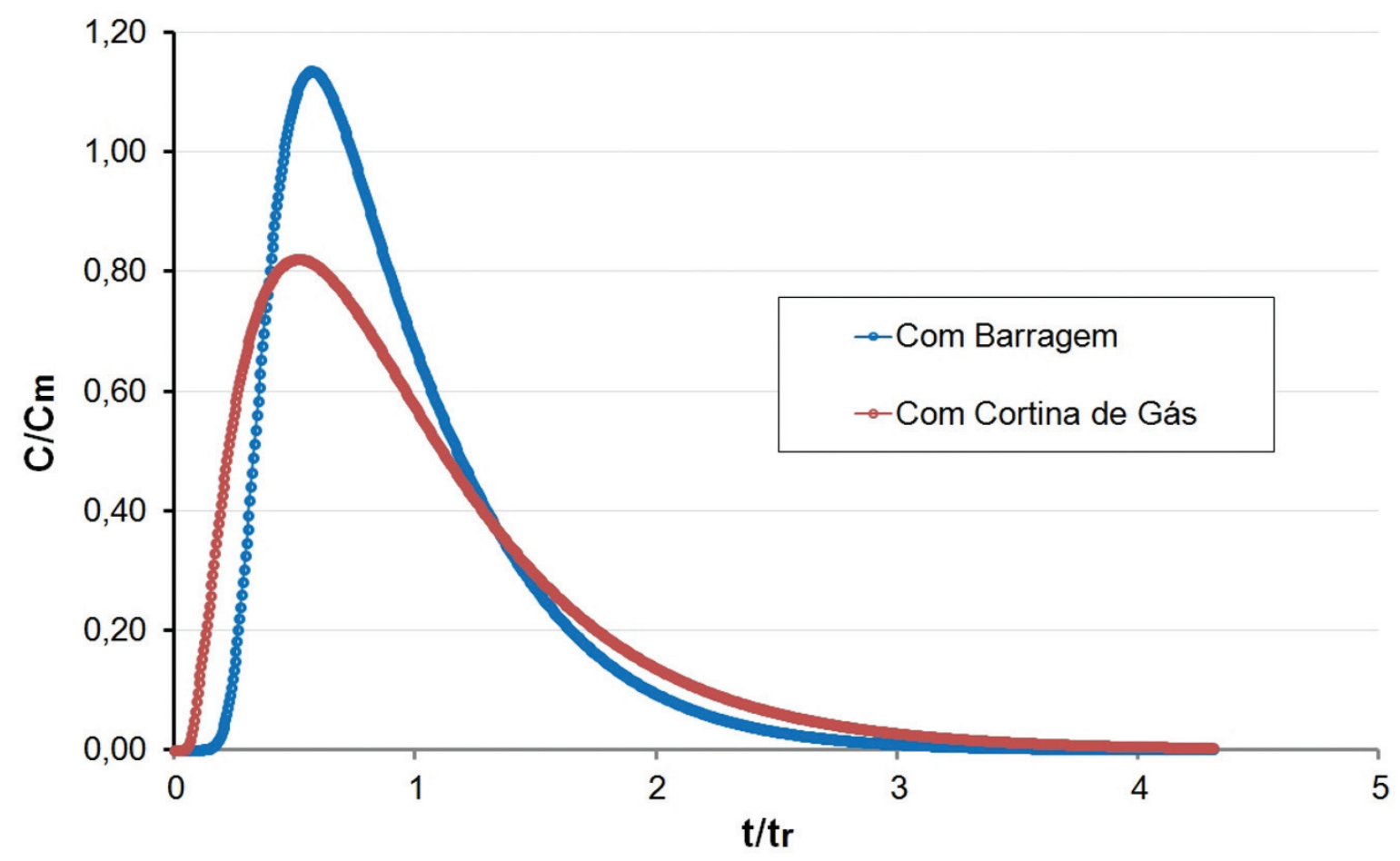

Figura 3. Comparação das curvas DTR obtidas nas simulações matemáticas do distribuidor sem injeção de gás e com injeção de gás.

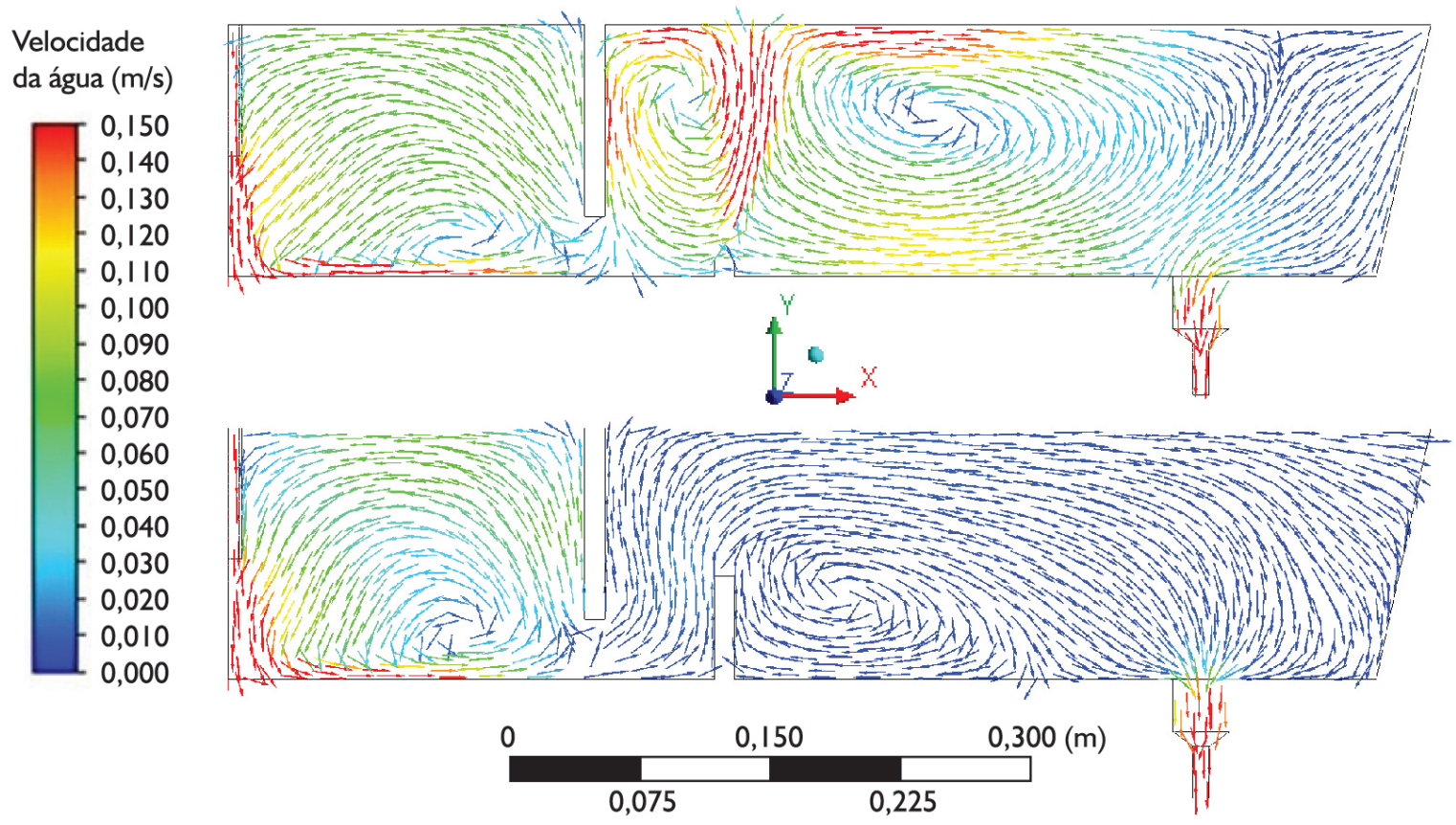

Figura 4. Comparação dos vetores de velocidade a partir de um corte longitudinal no modelo físico do distribuidor sem injeção de gás e com injeção de gás. 
ponto, o fluxo de água sofre alteração devido às diferentes configurações. Além disso, o fluxo da água é modificado pelas diferentes configurações empregadas. Nota-se que quando se substitui a barragem pela cortina de gás a distribuição de velocidades modifica e que ocorreu um aumento nos valores dos vetores de velocidade. Isto ocorreu pelo aumento da velocidade média da água proporcionada pela transferência de quantidade de movimento das bolhas de ar, sendo a configuração com injeção de gás a que levou às maiores velocidades. Apesar disso, não houve a diminuição da fração de volume morto no sistema quando se aplica o gás. A Figura 5 mostra a fração de volume de gás no plano de um corte transversal na seção de injeção de ar. Com a injeção de ar uma fina camada adjacente à parede tem uma

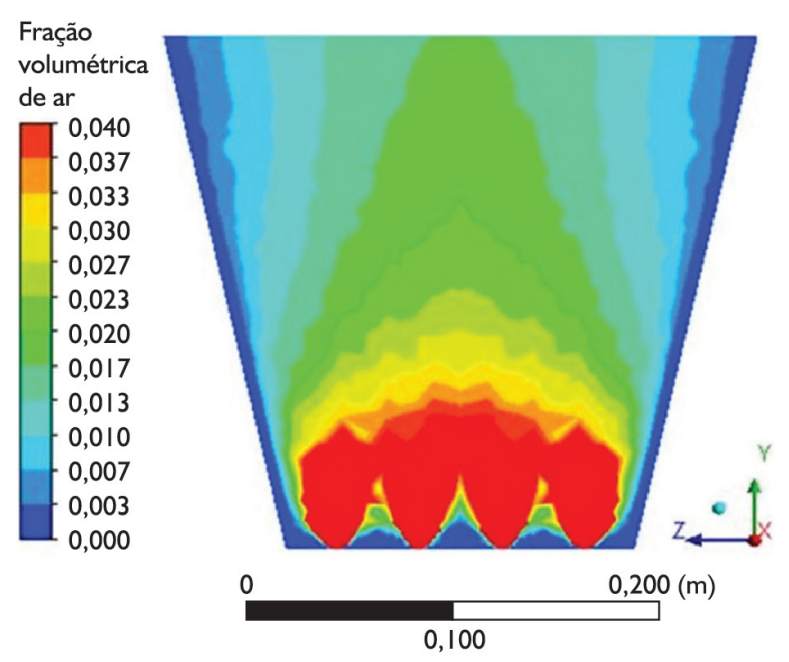

Figura 5. Fração de gás obtida na simulação matemática do modelo físico do distribuidor para a configuração com injeção de gás. passagem de água livre da interferência de ar. A barragem possui uma maior eficiência em conter e projetar o líquido para cima do que apenas a cortina de gás. No entanto, Zhong [4], Cwudzinski [5] e Neves [6] demonstraram que a aplicação da injeção de gás em conjunto com a barragem pode trazer benefícios para as propriedades fluidodinâmicas. No entanto, é preciso atenção na vazão de gás, pois altas vazões podem agitar a superfície do banho metálico e arrastar escória para o aço líquido.

\section{CONCLUSÃO}

Neste estudo procurou-se estudar o efeito da substituição da barragem pela cortina de gás. Analisando do ponto de vista das condições fluidodinâmicas, cada configuração possui sua vantagem, sendo que a configuração com a barragem possui menor fração de volume morto e maior fração de volume pistonado, enquanto que, o distribuidor com cortina de gás possui maior fração de volume de mistura perfeita. No entanto, tempo mínimo de residência e fração de volume pistonado muito baixos podem se relacionar à rápida saída do aço no distribuidor e consequentemente de arraste de inclusões para o molde. Desta forma, a escolha de uma ou outra configuração depende das condições que melhor se encaixam às práticas e propriedades metalúrgicas de produção de cada tipo de aço.

\section{Agradecimentos}

Ao Programa de Apoio à Pesquisa (PAPq) da Universidade do Estado de Minas Gerais (UEMG) pelo apoio e financiamento a esta pesquisa.

\section{REFERÊNCIAS}

I Szekely J, llegbusi OJ. The physical and mathematical modeling of tundish operations. New York: Editora SpringerVerlag; 1988.

2 Schade J, Smith MP, Palmer SE. Doubling Tundish Volume at AK Steel's Middletown Works: Structural criteria, design considerations end operating results. In Iron and Steel Society. Proceedings of the 79th Steelmaking Conference; 1996 March 24-27; Pittsburgh, PA. Warrendale, PA: I\&SM; 1996. p. 93-103.

3 Singh S, Koria SC. Tundish steel melt dynamics with and without flow modifiers through physical modeling. Ironmaking \& Steelmaking. 1996;23(3):225-263.

4 Zhong LC, Li LY, Wang B, Zhang L, Zhu LX, Zhang GF. Fluid flow behavior in slab continuous casting tundish with different configurations of gas bubbling curtain. Ironmaking \& Steelmaking. 2008;35(6):436-440. http://dx.doi. org/10.1 I79/174328108X318365.

5 Cwudzinski A. numerical simulation of liquid steel flow and behavior of non-metallic inclusions in one strand slab tundish with subflux turbulence controller and gas permeable barrier. Ironmaking \& Steelmaking. 2010;37(3):169I80. http://dx.doi.org/I0.1 179/030192309XI2549935902383.

6 Neves L. Modelamento matemático do escoamento multifásico no desgaseificador RH e no distribuidor de lingotamento contínuo [tese de doutorado]. Belo Horizonte: Universidade Federal de Minas Gerais; 2012. 
7 Paladino EE. Estudo do escoamento multifásico em medidores de pressão do tipo pressão diferencial [tese de doutorado]. Florianópolis: Universidade Federal de Santa Catarina; 2005.

8 Sahai Y, Emi T. Melt flow characterization in continuous casting tundishes. ISIJ International. 1996;36(6):667-672. http://dx.doi.org//0.2355/isijinternational.36.667.

Recebido em: 12 Ago. 2014

Aceito em: 25 Nov. 2014 